\title{
Empirical Evidence of Relationship between Virtual Lab Development and Students Learning through Field Trials on Vlab on Mechatronics
}

\author{
Tanuja Sheorey, Member, IACSIT
}

\begin{abstract}
Premier Institutes of technical education in India are currently involved in the development of virtual laboratories in almost all the engineering disciplines. These labs shall provide a platform for the students to conduct experiments in a virtual environment and enhance their capabilities. Extensive field trials are carried out. The study presents an analysis of feedback received on effectiveness of Virtual lab on Mechatronics. The results indicate that virtual experiments scale-up conceptual understanding and discovery based learning, unlike verification based conventional experiments. A properly designed virtual experiment of any category, with standard content, demonstration methodology and proper evaluation strategy can even replace real time experimentation.
\end{abstract}

Index Terms-Distance learning, field trials, ICT, simulation, virtual labs.

\section{INTRODUCTION}

Ministry of Human Resource Development (MHRD), Govt. of India under National Mission on Education through ICT (NMEICT) has given a project for setting up of virtual laboratories. The learners in the distance education system and those in remotely located educationally backward areas can avail the benefit of quality and relevant education, through ICT. These laboratories shall provide a platform for the students to conduct experiments in a virtual environment and enhance their capabilities. The Mission aims to provide infrastructural support for building the knowledge network among the institutions of higher education and seamlessly integrate it with the National Knowledge Network, by providing broadband connectivity. This would ensure free availability of e-content to all users / learners.

Performing experiments through Virtual laboratory (Vlab) is relatively new concept in technical education. The aim is to develop simulation based experiments through demonstration videos as productive learning tool similar to real time hands-on equipment. To ensure quality e-content, experiments of each Vlab are carefully designed and passed through an extensive review process. The emphasis from the start was on design / discovery based learning rather than verification based, as in conventional lab setups. Currently, field trials are being conducted by developers to gather information about "how students are benefitted from Vlabs?"

Manuscript received June 4, 2013; revised September 16, 2013.

Tanuja Sheorey is with PDPM Indian Institute of Technology, India (email: tanush@iiitdmj.ac.in). and "what makes an educationally effective Vlab[1], [2]?"

The study presents an analysis of feedback received on effectiveness of Virtual lab on Mechatronics. Experiments of a lab course on Mechatronics comprises of designing a system to carry out a particular task, or a control system to carry out task in a logical manner. Each experiment is having two major components. Designing of control logic circuit using simulation software and conducting experiment through hardware-in-loop simulation. Workshops are conducted across India and feedback is collected both from faculty and students.

Feedback results show that, 1 . Software simulation helps in understanding design of control logic in a better manner and 2. Videos of experiments on task-in-hand are the most effective as it provides sequence of logical flow of information through components [3]. The present study aims to establish empirical evidence about relationship between Vlab development and students learning.

\section{Vlab ON MEChATRONICS}

Experiments of a lab course on Mechatronics comprises of designing a system to carry out a particular task, or a control system to carry out task in a logical manner [4]. For example, an automatic car parking system needs designing of a logic circuit to fulfil the requirement of a parking facility. There are various ways to design the facility. In the first phase of virtual experiments, simulation of one possible design is shown that is fulfilling all the requirements. Logical flow of information is explained through algorithm. Students are encouraged to think about other possibilities of design. The simulation software is made available to them in open domain. This not only exposes learners to real-life industrial problem solving but also encourages them to design the logic circuit iteratively and come out with the best design/fail safe design. Each experiment is supported with photograph of individual components and of complete experiment setup to help student identify with hardware components. Detailed experiment procedure followed by experiment videos show performance of individual components according to designed logic.

All the experiments are application-oriented(taskbased).First phase begins with designing the system. Virtual experiments under mechatronics fall under two categories, I. Design based, involving design of a control system and verifying through hardware-in-loop simulation, and II. Enquiry based, where circuits are designed to perform experiments to enhance conceptualization via analysis of results. 
The second phase begins with assembly of all the hardware components as identified in design. Each hardware component is connected to control device, namely programmable logic controller (PLC) or microcontroller. Designed logic circuit will then be used to run hardware components, called hardware-in-loop simulation. The outcome of category I experiments is made available in the form of videos which shows performance of individual components according to designed logic. In category II experiments, outcome is given in terms of results of experiment and analysis of the results is shown to enhance conceptual learning.

\section{A. Design Based Virtual Experiments}

Designing of the experiment consists of making circuit diagram on available software having logical flow of information as desired. Validation of the design is carried out through software simulation under various conditions [5]. It helps in identifying with unwanted components, logical error in design, and better understanding of circuit logic. For example in designing a car parking facility, one should take care of logical flow of car arriving at the facility, checking for available parking slots and allotment of parking slot. A missing logic like "not taking into account cars leaving the facility" will bring error whereas parking slot is being allotted. Also, the design has to take care of cross checking like wrong parking, where suitable alarm signal should be raised to take care of the problem.

\section{B. Enquiry Based Virtual Experiments}

The task here is mostly to develop understanding of behaviour of components under varied conditions [3]. For example in designing a circuit to control speed of actuation of throttle check valve, behaviour of check valve needs to be understood completely. Whether task in hand requires meter-in or meter-out control, at what speed the actuation is to be done, etc. Equipped with the understanding, student can design hydraulic circuit on his own using appropriate controls.

\section{RESEARCH METHODOLOGY}

Computers have been in use to facilitate data acquisition, to provide real-time data display, to analyse these data, and to simulate complex phenomena. These methods have been shown to be effective to reduce the effort required and enhance accuracy with which the data can be processed.

The question now is "can software simulations and videos replace laboratory for hands-on equipment? Will it be as productive a learning tool as hands-on equipment, considering the importance of experiments in technical education [6]?

With technical education system, there is a growing awareness for ICT based education across Indian population. There is a growing need to put-in right effort for linking ICT based distance learning strategy with objectives of technical Institution, to enhance productive learning quality[4], [7], [8]. This includes both theory courses $\left(\right.$ NPTEL $\left.^{1}\right)$ and Vlab courses. This study is undertaken

\footnotetext{
${ }^{1}$ National Programme for Technology Enhanced Learning
}

within the context of ICT based virtual lab conduction, and attempts to provide the empirical evidence about the relationships between virtual lab development activities and student's performance.

Extensive field trials are being carried out by each developer with the aim of improving the quality of the contents and make a general comparison of various learning tools [1], [3], [6], [7]. Feedback workshops are conducted across India. Feedback form is prepared for a sample of students across the country to investigate the opinion of students regarding the effectiveness of concept understanding and be able to provide feel of real time experiment.

In general, feedback is obtained three ways: 1) From the students of the Institute to run Vlab as part of their curriculum (both undergraduate and post graduate), 2) Direct contact with students through feedback workshops across India, and 3) Students made to learn Vlab content before performing real life experiments (both from Institute and outside).

A pilot study was conducted by engaging 10 students of IIITDM Jabalpur. These students were learning the theory course simultaneously. When asked verbally about the importance of hands-on experiments, almost each of them replied, yes, "hands-on experiments are essential part of conceptual understanding". They were then given the Vlab experiment material to read and study before performing the experiment. There are three categories of students found as a result of pilot study. The students who have studied the material could design the logic circuit and run hardware-inloop simulation on their own. The students who have studied the material and taken up online quiz were able to comprehend the design logic in a better manner. When given the task of designing similar kind of circuit on simulation software, they were able to design quickly. The third category, though were able to cope up with the simulation software, could not finish the design of given logic circuit and nor the experiment.

Based on the feedback received during pilot study, feedback form was finalized to elicit respondents' opinions on the following [1], [6]:

- quality of the content

- conceptual understanding

- effectiveness of virtual lab through simulations

- completeness of the experiment w.r.t. its relation with theory

- Any other experiment to be covered under Vlab (to make it more comprehensive)

Feedback is mostly obtained through workshops. Feedback from faculty is collected who are engaged in teaching the relevant theory courses. Most of the teachers were satisfied when shown detailed experiment procedure, logic circuit algorithm and simulation. All of them gave $10 / 10$ to the effectiveness of the videos for understanding logical flow of information and making learning interesting. Unfortunately this feedback does not give us insight into whether videos help in visualizing the underlying problem and concepts which were ultimate goal of the experiments.

To select sample student candidates, list of technical Institutions of all categories offering a course on Mechatronics is made. From each category of Institutions, 
10 students are taken. Feedback from only those students is taken who have done theory course. The selected students were asked to study the material and take up online quiz. Responses obtained based on the verbal discussion and feedback received is summarized in the form of levels of difficulty encountered and ease of use.

\section{Level of Difficulty Encountered}

1) Some instructions are required before students could design their own logic circuit.

- The software used for simulating design of logic circuit is most commonly used in industrial practice. Students who have not undergone training may face initial difficulty. Sufficient background material is made available to learn it before venturing into design. Also, carefully studying the simulation will help in understanding "how" and "why" of the given design.

- The feedback obtained was mixed kind, depending on individual students training.

- For example, two of the responses from the students of the same Institute were, "we have studied the subject earlier and did not face any problem", and "the running of the software seemed to be bit complex and confusing".

2) Demo by developers or in-class demo was quite adequate for most of the students [3].

- Feedback was taken after a demo session, followed by a lab session.

- Since demo will not be possible with large student population targeted, algorithm has been explained in greater details.

- Some of the students gave feedback like"lengthy description", whereas the others praised the same owing to clarity in understanding.

- Another important feedback was "basic concepts are clear from the experiment and also we get innovative idea for such applications".

3) There should not be too much new information / concept given at once.

- For the same, common real-life problems were taken up with which students feel connected. Largely, the idea was accepted by the respondents.

- Care was taken in the first few experiments to divide the problem to avoid too many details.

- For example, in the experiment on speed control of DC motor using PWM, only micro controller based programming is explained and shown. Originally planned additional IC 555 based PWM is later dropped

4) The design circuit that might look impressive to an expert may be overwhelmingly complex for a student and may not result in useful understanding.

- In later experiments problems are taken up from industrial control, hence logic circuits are bound to become involved. Students are expected to understand them through detailed algorithm. Industrial control problems are involved ones and cannot be simplified beyond a limit else emphasis on the learning goals would be lost.

- For example, in the design of car parking facility, gate should always get opened when a car is leaving, but it need not be true for an arriving car because that needs availability of vacant slot. There is a single motor to be actuated for both the purpose and hence a flag needs to be used to separate both the functions.

- This logic is explained along with algorithm.

- If a student learns this concept carefully, he may utilize the same in other problems as well in the right context.

5) Eliminate all potential distractions and emphasize only those items that are pedagogically important [3].

- It might enhance cognitive load on students and bring confusion.

- Students are sometimes unable to identify some features that otherwise goes unnoticed by experts.

- For example in designing of automatic sorting system, three LEDs of different colours are used to depict sorting of three different kinds of item. The purpose was to install them at the location of manager to get the signal in case any of the line is not operating properly or got struck due to some problems. In factit was meant for a fail-safe design. In the absence of proper understanding of the concept, student might try to relate it to sorting and get confused.

\section{Level of Ease}

1) Though for the sake of completeness, all aspects of experiment are described in large details, the obvious benefit of hardware-in-loop simulation is the visual model that is provided for the students. It is far simpler and reliable to comprehend something by seeing instead of telling or describing it in the text mode.

- Almost all the respondents liked the idea of teaching concepts through real life applications and demonstrating them through videos that are easily interpretable [3].

- Easy interpretation of the problem and step by step procedure is liked by many.

- For example, in the car parking facility, student can easily visualize what should be the action when a car is wrongly parked; though this aspect was not the main issue, but without this the design would have failed. Such hidden design issues need to be addressed and explained to make a design fail-safe.

2) As far as possible a common structure has been provided to all the Vlab experiments.

- Each experiment is written with the same structure as is used in conventional setup.

- Students feel comfortable in locating the desired details, whether it is circuit diagram or experiment setup, videos, etc.

3) Consistent representation of the objects appearing in more than one experiment.

- Student might perceive the objects different ones.

- In Vlabs on Mechatronics, standard symbols are used to represent objects.

4) Experiments were arranged in a manner where they begin with most simple features. With less number of control parameters or simple control loop, students will remain engaged in exploring logical flow that ultimately lead to conceptual learning.

- For example in designing inventory control system, care is taken not to indulge in detailing of inventory arrangement or ordering system. Instead, emphasis 
was on how to get inventory automatically through sensor system and raise the signal if ordering is needed for a particular item.

5) Pictures of individual components as well as of complete setup are given in the form of easily downloadable image format.

- Identification with component details becomes easier and effective.

6) Students relate things easily by colours.

- In experiments on pneumatic and hydraulic controls, different colour code is used for flow of air/oil (by blue) and electricity (red).

During the feedback workshops conducted at the place where lab facility is available, two questionswere additionally asked to bring out effectiveness of Vlab clearly:

1) Should simulation based experiments be used only when costly equipments are not affordable or not available in the laboratory?

Answer given by respondents was, no. Conventional experiments are mostly verification based andnot always meant for enhancing their conceptual understanding.
2) Should it be used as essential part of conceptual learning prior to performing real-life experiments, wherever possible?"

The answer given by respondents was, yes. Software simulation, followed by hardware-in-loop simulation provides complete understanding of the topic / task in hand. When given a different task to perform experiment, it was easier to design logic circuit and connect various components to perform full experiment.

\section{OUTCOME}

The Vlabs stressed discovery rather than verification and included elements of "industrial control system design". All the experiments are application oriented. The goal of the experiments was for students to develop an understanding of logic circuits, the requirement of components for designing a logic system for a task-in-hand, and the skills associated with connecting all the components and testing the system for logical behaviour (hardware-in-loop simulation).

TABLE I: RESPONSES RECEIVED DURING FEEDBACK WORKSHOP

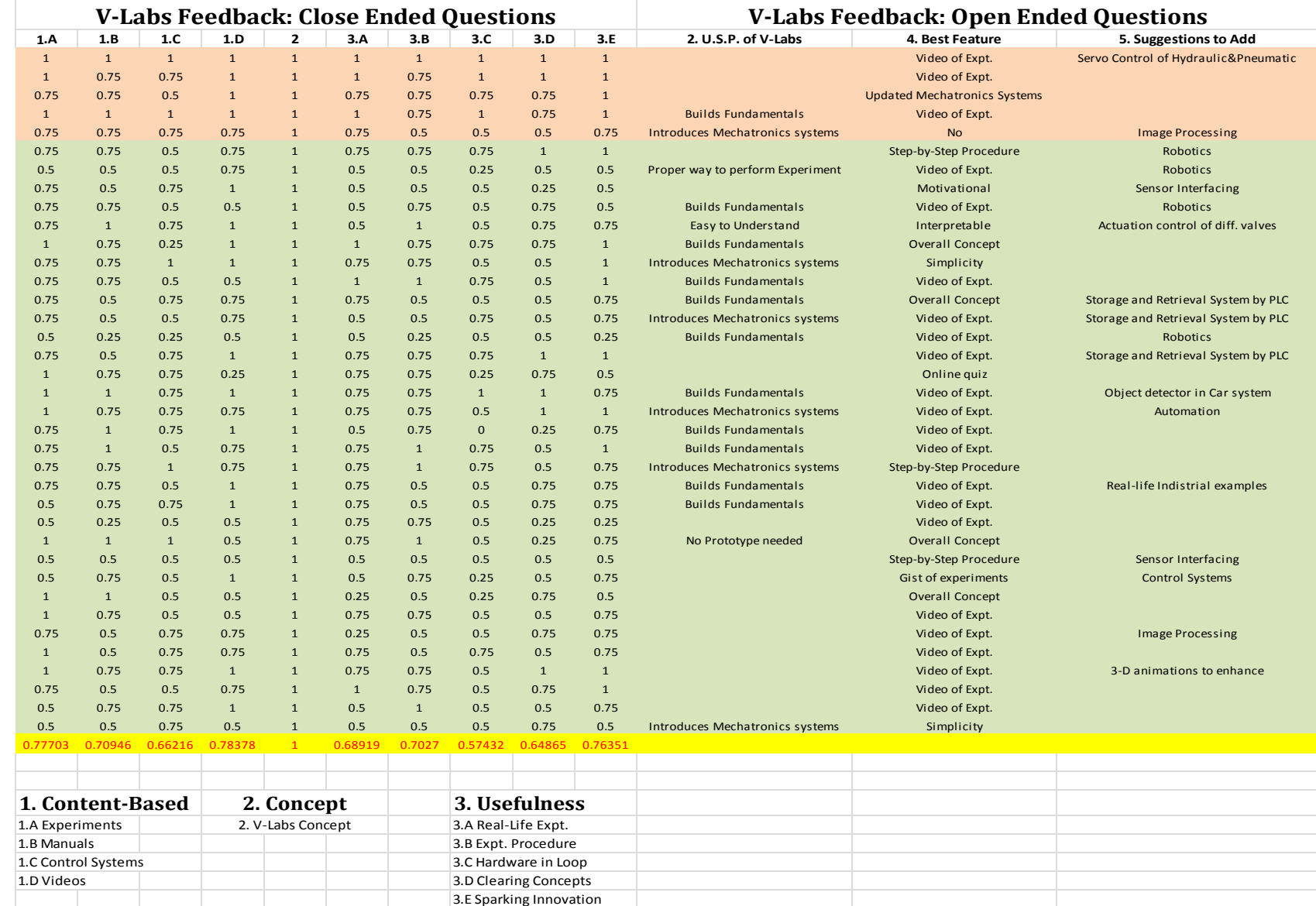

At the end of each laboratory students are given online self-evaluation. It comprises of testing of background theory, procedural details and design of logic for the particular task. In addition, testing of knowledge about circuit design, hardware components, flow of logic, etc, is also included. The questions on logic circuit help in strengthening concept understanding through "how", "why", "what", etc. Examples given below illustrate the point:

1) "Why Q2 (output signal representing retraction of DAC) is latched at the end of the program?. The answer is to unclamp the job already finished and not to represent end of cycle."; Or

2) What will be the outcome if instead of internal marker M1, timer T1 is reset? The answer is actuator will continuously extend and retract even without loading of any goods, because M1 will remain set and causes unwanted actuation.

Encouraged by pilot study performed on our own students, 
feedback form was finalized and field trials are conducted in the form of workshops. Feedback forms were analysed point-wise. Analysis of closed questions and open-ended questions are done separately. About 50 usable responses were considered out of about 200 forms received.

Table I shows the responses received from 40 students on questions 1 through 5. Average of 40 responses for each question is given on the last row. Question 1 is having 4 parts as listed in the Table. For example on the question of "simulations were informative", average is around 0.77 , indicating most of the responses were between excellent and very good. But on the question of "the control system simulations were easily interpretable", mostly responses fell in between very good and good category (0.66). The reason can be attributed to not giving enough time to understand logic circuit.

Question 2 was most important from the point of view of gathering evidence on understanding of basic concepts of a course through Vlab [6]. It was an open ended question. Answer of almost all the respondents was YES(including those who have not given other responses seriously and hence not included). In addition, table also lists the comments given by most of the respondents, pointing to basic concept learning (USP of Vlabs).

Question 3 again is having 5 parts. On the question of "could you relate hardware-in-loop simulation (HIL)", average is around 0.57 , indicating most of the responses fell in good category. The reason here is not able to understand the term HIL. Because answer to open ended question 4 "the most interesting feature of Vlab", most of the respondents replied "video of experiment" that incorporate HIL.

Question 5 is again an open ended one and number of request are received for development of additional experiments on rather diverted topics like robotics, controls, etc. Some of the respondents actually asked for inclusion of more involved industrial projects to make the learning interesting.

Results of study indicate that properly designed simulations used in the right contexts can be more effective educational tools than real laboratory equipment; both in developing student interest with real equipment and at fostering student conceptual understanding. In this particular environment, given an industrial control design problem with complete description and the explicit goals, students were able to design logic circuits, identify component requirement and acquire skill at manipulating these components and bring-out final control system.

It is important to point out here that concept of Vlab is learning anytime, from anywhere. The feedbacks analysed in the present study are received immediately after demo and lab session. Respondents were not given chance to study experiments on their convenience. Also imperative here is to point out that grasping level of students is varied and the fact that Vlabs are meant for a large student population, care is taken to provide additional details for the benefit of slowest learner.

In one of the earlier feedback received, respondent opines that Vlab is extremely helpful for the ones who want to upgrade themselves in order to contribute in their chosen field. As the content can be downloaded and be studied anytime, there is ample scope of self-improvement. The response is pointing to the very essence of Vlab concept. Any person who wants to learn should not have barrier of the classroom and association with an Institute. Author's team is developing additional experiments on the topics suggested in the feedback. Also field trials are still undergoing. To make the feedback comprehensive, informal learners who are not associated with formal curriculum could be targeted next, through online survey.

\section{CONCLUSION}

Owing to experiments being application oriented, it was found that students remain engaged in learning. Further, number of requests received for development of experiment on rather diverted topics proves that Vlabs provide better conceptual learning environment. The students welcomed the task based experiments to design a virtual system and found them to be more productive.

Feedback trials are still undergoing. Based on verbal discussions and earlier feedback, this work pointed out many issues (Sec 3.A and 3.B), if not considered while developing experiment, may result in Vlabs not achieving educational effectiveness [6]. A properly designed virtual experiment of any category, with standard content, demonstration methodology and proper evaluation strategy can even replace real time experimentation [5], [8]. It also brings in the benefit of enhanced conceptual understanding and requisite hands-on experience. However, the concept of virtual laboratory is still new and more research should go into designing the content and evaluation procedure based on feedback and field trials.

\section{ACKNOWLEDGMENT}

I would like to thank Mr. Arpit Gupta who has helped in conducting feedback workshop as well as compiling the data for analysis. This work was conducted with the support of Ministry of Human Resource and Development under National Mission on Education through Information and Communication Technology.

\section{REFERENCES}

[1] R. Hashim, H. Ahmed, and C. Z. Abdullah, "Antecedents of ICT of distance learning education students," TOJ"T: Turkish online journal of educational technology, vol. 9, issue 1, January, 2010.

[2] T. Plomp, W. J. Pelgrum, and N. Law, "SITES2006-International comparative survey of pedagogical practices and ICT in education," Educ Inf Technol, vol. 12, pp. 83-92, 2007.

[3] W. K. Adams et al., "A study of educational simulations Part II Interface design," University of Colorado, Physics Education Technology Web site.

[4] T. Sheorey and V. K. Gupta, "Effective Virtual Laboratory Content Generation and Accessibility for Enhanced Skill Development through ICT," presented at 3rd ICDLE conference at Singapore, 2011.

[5] N. D. Finkelstein et al., "When learning about the real world is better done virtually: A study of substituting computer simulations for laboratory equipment," Physical review special topics, Phtsics education research $1,010103,2005$.

[6] A. Tracy et al., "Virtual labs in the online biology course: Student perceptions of effectiveness and usability," MERLOT journal of online learning and teaching, vol. 3, no. 2, June 2007. 
[7] B. Foss, B. F. Oftedal, and A. Løkken, "Rich Media e-Compendiums: A New Tool for Enhanced Learning in Higher Education," European Journal of Open, Distance and e-Learning, vol. 16, no. 1, pp. 105, 2013.

[8] H. Ndahi, "The Use of Innovative Methods to Deliver Technology Education Laboratory Courses via Distance Learning: A Strategy to Increase Enrollment," Journal of Technology Education, vol. 17, no. 2, Spring 2006.
Tanuja Sheore was born at Jabalpur, Madhya Pradesh, India on June 6, 1963, who has earned BE (Mechanical Engineering) from Government Engineering College, Jabalpur in 1985, M. Tech. (Thermal) from M. A

National Institute of Technology, Bhopal in 1988 and Doctorate from IIT Kanpur in 2002. Throughout the carrier, she has taught various professional courses of mechanical engineering. Her research interests are in applied fields like renewable energy sources, automation, microfluidics and biomedical devices. She is presently Professor of Mechanical engineering at PDPM Indian Institute of Technology, Design and Manufacturing Jabalpur and handling a couple of funded projects one among this is funded by MHRD, Govt. of India namely "development of virtual laboratory on Mechatronics". A fully functional laboratory has been established in the Institute by her team. 